\title{
Is the Early Universe Fractal?
}

\author{
E. M. de Gouveia Dal Pino ${ }^{1}$, A. Hetem ${ }^{1}$, J. E. Horvath ${ }^{1}$, \& T. Villela ${ }^{2}$ \\ 1. Universidade de São Paulo, IAG, São Paulo, SP 9638, 01065-970, Brazil \\ 2. INPE, São José dos Campos, SP, 12201-970, Brazil
}

\section{Introduction}

It is generally believed that on very large scales the distribution of matter in the universe is homogeneous and effectively all the existing theoretical approaches are based on this assumption. However, recent re-analysis of galaxy-galaxy and cluster-cluster correlations by Coleman and Pietronero (1992, hereafter CP) and Luo and Schramm (1992, hereafter LS) indicates that the distribution of the visible matter in the universe is fractal or multifractal up to the present observed limits $\left(\sim 100 \mathrm{~h}^{-1} \mathrm{Mpc}\right.$ for $\mathrm{H}_{\mathrm{O}}=100 \mathrm{~h} \mathrm{~km} \mathrm{~s}^{-1} \mathrm{Mpc}^{-1}$ and $\left.0.5 \leq \mathrm{h} \leq 1\right)$ without any evidence for homogenization on those scales. The fractal dimension obtained from these analyses is $D$ 1.2 - 1.3 (CP; LS).

The temperature fluctuations of about $13 \mu \mathrm{K}$ recently detected by the COBE team in the 2.7 $\mathrm{K}$ cosmic microwave background radiation (CMBR) over angular scales larger than $7^{\circ}$ (e.g., Smoot et al. 1992) correspond to linear sizes of $\sim 1000 \mathrm{~h}^{-1} \mathrm{Mpc}$. According to the Sachs-Wolfe effect, they were imprinted on the CMBR by primordial density fluctuations of the matter at the recombination layer and thus, provide a signature of the seeds of the structures observed in the present universe. In this work, we analyse these temperature fluctuations and show that they are also consistent with a fractal structure with fractal dimension $\mathrm{D}=1.43 \pm 0.07$.

\section{The Fractal Analysis}

A fractal consists of a system in which more and more structure appears at smaller and smaller scales and the structure at small scales is similar to the one at large scales. The relation between the perimeter $\mathrm{p}$ and the area a of a two-dimensional fractal distribution is (e.g., Gouveia Dal Pino et al. 1994): $a^{1 / 2}=F p^{1 / D}$, where $D$ is the fractal dimension and $F$ is the shape factor which is related to the form of the distribution. For fractals, $D$ is in the range $1<D<2$.

To obtain the fractal dimension from the CMBR image (Smoot et al. 1992), we measured the perimeter and the area of regions situated within iso-temperature contours (Gouveia Dal Pino et al. 1994). The points in the logarithmic plot of perimeter versus area, obtained from the various temperature contours of the distribution, are fitted by a straight line where $D / 2$ is the slope, in agreement with the equation above. The result is shown in Fig. 1. The corresponding fractal dimension is $\mathrm{D}=1.43 \pm 0.07$ and the shape factor is $\mathrm{F}=0.42 \pm 0.04$.

The fractal dimension evaluated above is in reasonable agreement with the fractal dimension evaluated by CP and LS from galaxy-galaxy and cluster-cluster correlations up to $\sim 100$ $\mathrm{h}^{-1} \mathrm{Mpc}$. The fact that the observed temperature fluctuations correspond to scales $\gg 100 \mathrm{~h}^{-1}$ $\mathrm{Mpc}$ and are signatures of primordial density fluctuations suggests that the structure of the matter at the early universe was already fractal and thus non-homogeneous on those scales.

If we accept the results above, we can inquire if the fractal structure imprinted on the CMBR by the density fluctuations at $t_{r e c}$, on scales $\gg 100 \mathrm{~h}^{-1} \mathrm{Mpc}$, still persists at the present large scale universe as it seems to occur on smaller scales. If so, how far does the fractal correlation extend or where does the universe become effectively homogeneous? We can also argue if the observed fractal structure on large scales at $t_{\text {rec }}$ also occurs on small scales at that epoch. We certainly do not have the answers to all these questions but we can try to make some tentative predictions. 
Let us borrow from LS, the assumption that some kind of growth process provides the fractal correlation while gravity enhances the correlation amplitude on small scales. Considering some initial primordial seeds (or density fluctuations), the growth process will provide the

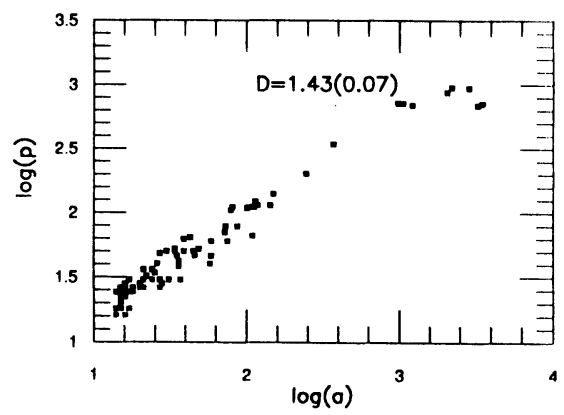

Figure 1. Perimeter versus area relation obtained from the temperature contours of the observed distribution of the CMBR fluctuations.

aggregation of matter to the seeds, and the growth rate is limited by the diffusing flux of matter onto them. Ball and Witten (1984) find that the fractal grown from a diffusion-limited process implies that $\mathrm{D} \geq \mathrm{d}-1$, where $\mathrm{d}$ is the dimension of the growth space of the aggregate. In the present work, the derived fractal dimension $\mathrm{D} \approx 1.4$ results $\mathrm{d}<2.4$. In other words, the growth space should involve a 2-dimensional sheetlike object and favor, for example, light domain wall, pancake, or cosmic string seed models (see, e.g., LS). Since the growth process is limited by the diffusion of particles onto the aggregate, its rate can become smaller than the expansion rate of the universe at some extension. At this point we should expect the end of the fractal structure in the universe and the beginning of homogeneity. To evaluate the breakdown scale of the fractal correlation (L), LS assume that the aggregation of matter onto the seeds, during the growth of a fractal up to $\mathrm{L}$, will perturb the CMBR up to the observed maximum amplitude $\delta \mathrm{T} / \mathrm{T} \sim 10^{-5}$. Then, they find a lower limit for the fractal correlation length $\mathrm{L} \geq 100 \mathrm{~h}^{-1} \mathrm{Mpc}$. If correct, this result indicates that the fractal structure obtained in the present work for primordial seeds at large scales $\left(>1000 \mathrm{~h}^{-1} \mathrm{Mpc}\right.$ ) has probably been diluted as a consequence of the diffusion-limited growth process in the expanding universe. However, further observations on different angular scales are still required to tell us the real value of $L$ and thus, where the fractal scale ends up and homogeneity begins.

Finally, the cosmological principle implies the condition of isotropy and homogeneity. But, according to the results of the present work (and also of CP and LS), this assumption is not supported since the distribution of matter in the present visible universe on scales up to $\sim 100 \mathrm{~h}^{-1}$ $\mathrm{Mpc}$ and also the distribution in the early universe on scales $>1000 \mathrm{~h}^{-1} \mathrm{Mpc}$, appear to be fractal. As pointed out by CP, the fractal structure implies an asymmetry between space points occupied by the structure and empty points and suggests a modification of the cosmological principle: in $a$ simple fractal structure, from every point of the system one observes statistically the same kind of local overdensity characterized by a single exponent $D$. Clearly, this modified cosmological principle is still satisfactory and does not imply a preferred point in the universe.

\section{References}

Ball, R.C. and Witten, T.A. 1984, Phys. Rev. A29, 2966

Coleman, P.H. \& Pietronero, L. 1992, Phys. Reps., 213, 311 (CP).

Gouveia Dal Pino, E.M., et al. 1994, Ap. J. (submitted)

Luo, X. \& Schramm, D.N. 1992, Science, 256, 513.

Smoot, G.F. et al. 1992, Ap.J., 396, L1. 